\title{
Effect of Particle Size and Ligand on the Tribological Properties of Amino Functionalized Hairy Silica Nanoparticles as an Additive to Polyalphaolefin
}

\author{
Tianyi Sui, Baoyu Song, Feng Zhang, and Qingxiang Yang \\ School of Mechatronics Engineering, Harbin Institute of Technology, Harbin, Heilongjiang 150001, China \\ Correspondence should be addressed to Baoyu Song; sby@hit.edu.cn
}

Received 25 August 2015; Accepted 7 December 2015

Academic Editor: Jean M. Greneche

Copyright (C) 2015 Tianyi Sui et al. This is an open access article distributed under the Creative Commons Attribution License, which permits unrestricted use, distribution, and reproduction in any medium, provided the original work is properly cited.

Hairy nanoparticles, which graft organic chains on nanoparticles, have led to a wide variety of advanced materials and have been applied in many fields over the past two decades. In this paper, effects of nanoparticle size and organic chain on the tribological properties of amino functionalized hairy silica nanoparticles (HSNs) were investigated. Silica nanoparticles with different sizes and amino group organic chains were synthesized and dispersed into polyalphaolefin (PAO) via a modified process. The synthesized HSNs were characterized by variety of methods. The tribology properties of those HSNs were investigated using a four-ball tribometer. The coefficient of friction and wear scar diameter were measured and analyzed. It was found that the HSNs could form a stable homogeneous solution with PAO. The tribological performance of the PAO 100 was enhanced dramatically by adding the HSNs. The data suggested that HSNs with larger size, longer organic chains, and more amino groups gave better antiwear and friction reduction properties than other nanoparticles.

\section{Introduction}

During the past three decades, increasingly efforts were devoted to the study of organic-inorganic nanoparticles, and those nanoparticles were explored for numerous fields of applications such as optical [1], medical [2], biological [3], and rheological field [4] due to their unique advance properties. Among these properties of organic-inorganic nanoparticles, their antiwear and friction reduction properties are those of highest interest. By covering organic chains on the surface of inorganic nanoparticles, the dispersity and stability of inorganic nanoparticles in organic solvent would increase significantly, especially in lubricants. Varieties of nanoparticles, such as $\mathrm{TiO}_{2}[5,6], \mathrm{CuO}[7,8], \mathrm{ZnO}[9], \mathrm{Fe}_{3} \mathrm{O}_{4}[10], \mathrm{Sn}$ [11], and $\mathrm{WS}_{2}$ [12], were added to lubricants as additives and those nanoparticles exhibit good tribological performances. Among these nanoparticles, silica nanoparticles are particularly attractive because of their good environmental compatibility, low cost, and excellent tribological performance. Silica nanoparticles were modified with variety of ligands [13-17], and amino functionalized silica nanoparticles were one of the best hairy silica nanoparticles to be applied in tribology field due to their special properties: silanes with primary amino functional groups show good compatibility with organic solvents $[18,19]$, form amine-metal bonds with metallic substrates [20], and provide reactive groups which could be further functionalized by chemicals [21-23]. Those properties make the amino silane interesting and promising modifier which could improve the tribological property of silica nanoparticles and enhance their stability in lubricants [18]. Amino functionalized silica nanoparticles with different chain lengths and amino group numbers were studied with their tribological properties. However, limited research focused on the effect of chain length and amino group number on the tribological properties of silica nanoparticles. Silica nanoparticles with diameters of $12 \mathrm{~nm}[24,25], 20 \mathrm{~nm}$ $[18,26], 40 \mathrm{~nm}$ [19], and $92 \mathrm{~nm}$ [27] were reported to exhibit good antiwear and friction reduction properties when adding to lubricants or filling in composites. However, no research focused on the effect of nanoscale $(<100 \mathrm{~nm})$ particle size on the tribological properties of silica nanoparticles, and the influence of particle size on the antiwear and friction reduction performance remains unclear. 


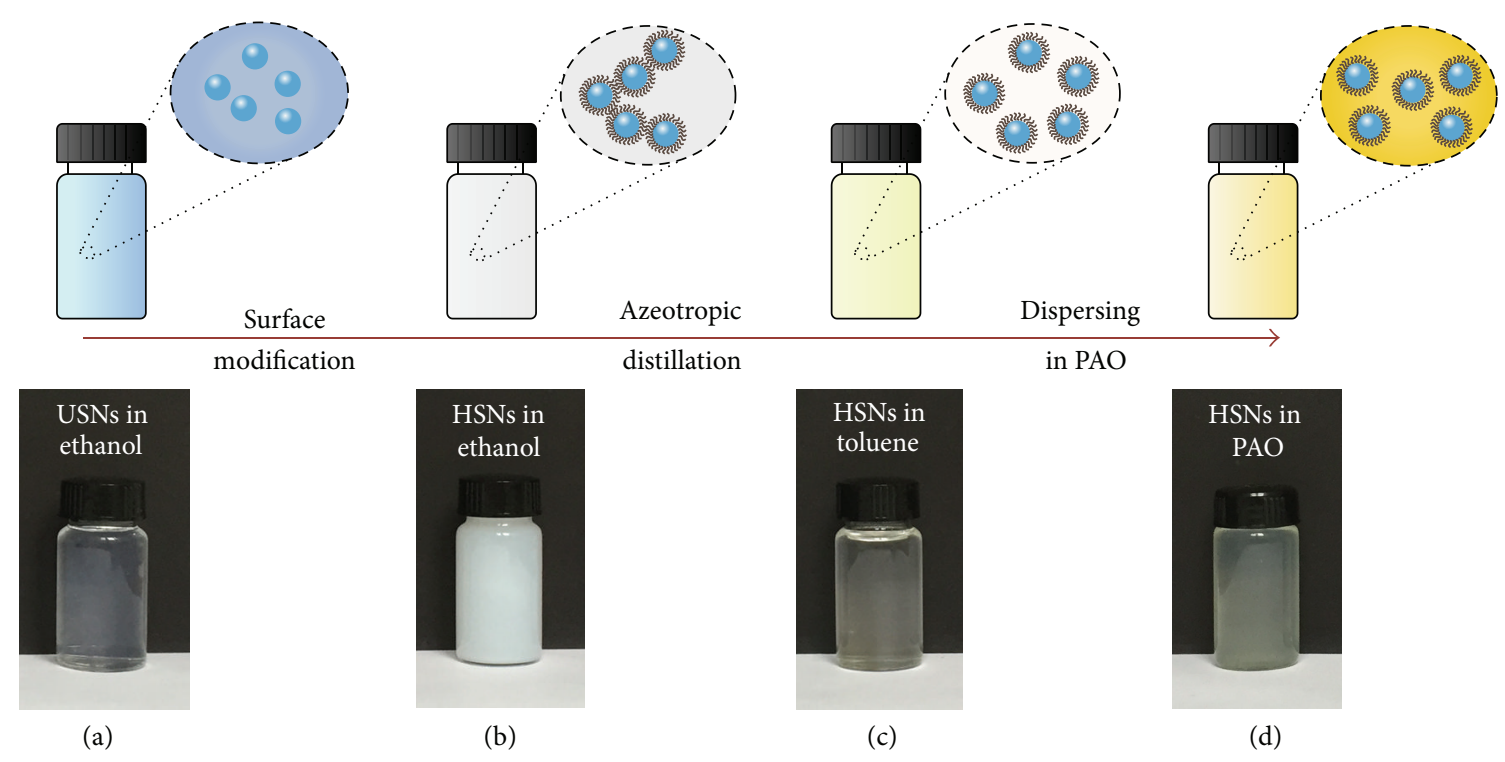

FIGURE 1: Synthesis and dispersion of HSNs. (a) USNs dispersed in ethanol. (b) HSNs dispersed in ethanol. (c) HSNs dispersed in toluene. (d) HSNs dispersed in PAO.

Keeping in view previous work reviewed above, silica nanoparticles with wide range of diameters were tested with their tribological performances [18, 19, 24-27]; those nanoparticles show good antiwear and friction reduction properties when adding to lubricant, but no work studied the influence of nanoscale particle size $(<100 \mathrm{~nm})$ on the tribological properties of HSNs. Some papers studied the tribological properties of amino functionalized nanomaterials $[18,21-$ $23,25]$, but no work focused on the effect of chain length and amino functional group on the tribological performance of silica nanoparticles as lubricant additives. The influences of amino functionalized organic chain and nanoparticle size on the tribological performance of HSNs are valuable to be studied for further understanding the antiwear and friction reduction properties of HSNs as lubricant additives.

In this paper, HSNs with different sizes and amino organic chains were synthesized based on Stöber method $[19,28]$ and characterized by various methods such as SEM, FTIR, and TGA. Those nanoparticles were dispersed in polyalphaolefin (PAO) 100 lubricants with different concentration. The HSNsPAO 100 lubricants were tested using a four-ball tribometer. The optimal particle size, concentration, and amino organic chain for silica nanoparticles were investigated. Based on the test result, the antifriction mechanisms of HSNs were discussed.

\section{Experimental Section}

2.1. Preparation of HSNs. Figure 1 shows a schematic diagram of preparation process of PAO-HSNs lubricant, including four main steps as preparation of USNs, surface modification of USNs, solvent transfer of HSNs, and dispersion of HSNs in lubricant.

USNs were prepared using Stöber method. The tetraethyl orthosilicate (TEOS) was added to the mixture of ethanol, water, and aqueous ammonia and the resulting mixture was gently stirred at $400 \mathrm{RPM}$ at temperature of $40^{\circ} \mathrm{C}$. In this paper, three sizes of silica nanoparticles $(20 \mathrm{~nm}, 50 \mathrm{~nm}$, and $100 \mathrm{~nm}$ ) were prepared and further modified in the experiment. Different sizes of nanoparticles were obtained by varying the concentrations of water, aqueous ammonia, and TEOS. For $100 \mathrm{~nm}$ silica nanoparticles, the mixture of ethanol, water, and aqueous ammonia was prepared by adding $2 \mathrm{wt} \%$ of water and $5 \mathrm{wt} \%$ of aqueous ammonia to ethanol. After the mixture was heated to $40^{\circ} \mathrm{C}, 5 \mathrm{wt} \%$ TEOS was added to it by one time. After 3 hours, the solution became slightly turbid and blue, which indicated the formation of $100 \mathrm{~nm}$ USNs. For $50 \mathrm{~nm}$ silica nanoparticles, the synthesis process was the same as that for $100 \mathrm{~nm}$ particles while the concentration of aqueous ammonia was changed to $3 \mathrm{wt} \%$. For $20 \mathrm{~nm}$ silica nanoparticles, the concentrations of ethanol, water, and aqueous ammonia were $2 \mathrm{wt} \%$, $3 \mathrm{wt} \%$, and $5 \mathrm{wt} \%$, the TEOS was divided into two aliquots, and the second aliquot was added to the mixture after 3 hours of the first aliquots. After the second aliquot was added, the mixture was stirred at 400 RPM and heated for 3 hours. As shown in Figure 1(a), the solution became clear and slight blue after the reaction, which indicates that silica nanoparticles were monodispersed with narrow size distribution.

The USNs synthesized above were further modified by tethering organic silanes onto them. Three kinds of amino functionalized silanes, (3-aminopropyl)trimethoxysilane (APS), [3-(2-aminoethyl amino)propyl]trimethoxysilane (AEAPS), and N1-(3-trimethoxysilylpropyl)diethylenetriamine (DETAS), were purchased from Sigma-Aldrich Chemicals and used as received. Before the linking reaction, the silica nanoparticles were purified by dialysis in ethanol using a 10,000 MWCO snakeskin dialysis tube bought from Thermo Scientific. Unreacted TEOS was removed after 


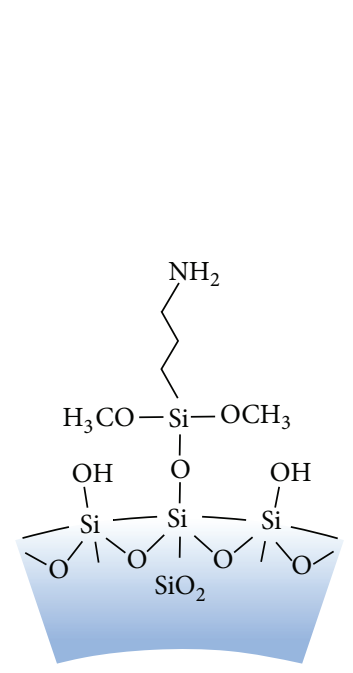

(a)

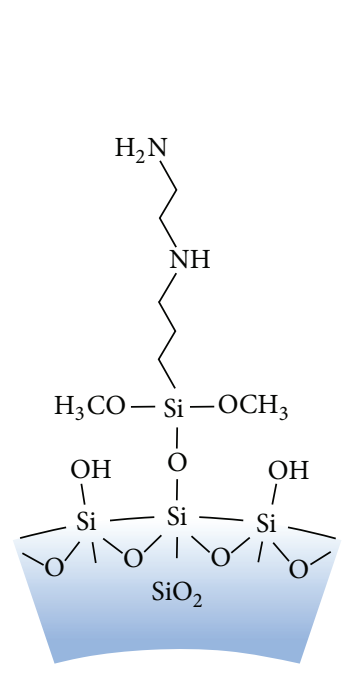

(b)

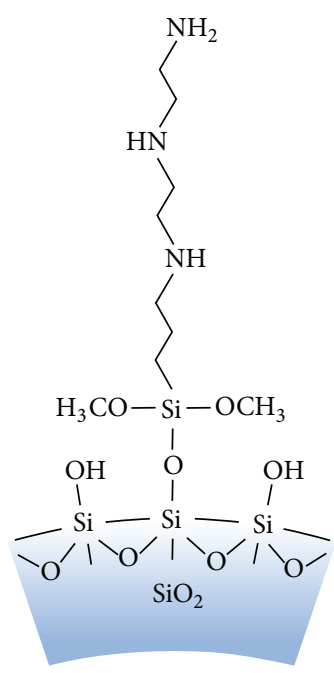

(c)

FIgURE 2: Molecular structures of (a) APS modified HSNs, (b) AEAPS modified HSNs, and (c) DETAS modified HSNs.

the purification and USNs solution was obtained. The silane was then added to the USNs solution drop by drop with rapid stirring at 600 RPM and gently stirred at 300 RPM for $12 \mathrm{~h}$ at $50^{\circ} \mathrm{C}$. After the linking reaction, the silica nanoparticles were dialyzed in ethanol using 10,000 MWCO snake skin dialysis tube to remove the unlinked silane and impurities. The HSNs-ethanol solution was obtained after the purification process. As shown in Figure 1(b), the HSNs-ethanol solution became white and cloudy, which suggested that nanoparticles formed micelles in the ethanol solution. Figure 2 shows the molecular structures of HSNs with different silanes. After linking reaction, the covalent siloxane bridges were created between siloxane and hydroxyl group on the surface of the silica nanoparticle. The surface properties were changed with the appearance of the organic chains on inorganic nanoparticle surface, which make it possible to disperse nanoparticles and form homogeneous solution in organic solvent.

2.2. Preparation of HSNs-PAO Lubricants. To test the tribology performance of HSNs as lubricant additive, HSNs were dispersed in PAO 100 purchased from Shanghai Fox Chemical Technology. Before dispersing them into PAO, silica nanoparticles were first transferred from ethanol to toluene. Azeotropic distillation was applied in this process in order to transfer silica nanoparticles from ethanol to toluene while it kept nanoparticles dispersed in the whole process without any aggregation. Toluene was added to the HSNs-ethanol solution obtained above to form a 3/1 mixture of toluene and ethanol. The mixture was then kept at $70^{\circ} \mathrm{C}$ to remove the ethanol. HSNs-toluene solution was obtained after the ethanol was driven off. As shown in Figure 1(c), HSNs-toluene solution was homogeneous with excellent optical transparency, which suggested that the HSNs were dispersed well in the solvent. The solution was then added to PAO with fast stirring. After that, the mixture was transferred to oven to remove the toluene. As shown in
Figure 1(d), HSNs formed homogeneous stable solution with PAO 100.

In the preparation process of HSNs-PAO lubricants, USNs were modified with silanes as soon as their synthesis and purification process finished, which could reduce the aggregation between silica nanoparticles. After the modification, the HSNs were purified by dialysis in ethanol, transferred from ethanol to toluene by azeotropic distillation and then dispersed into PAO lubricants. During the whole preparation process, HSNs were kept dispersed in solvent without aggregation and redispersion, which could help enhancing the stability and dispersity of nanoparticles in lubricants.

2.3. Characterization. A scanning electron microscope (SEM) Hitachi SU8010 was used to characterize the morphology of silica nanoparticles. Before the experiment, silica nanoparticles were treated with sputter-gold to enhance the conductivity. Surface areas of USNs were measured by Brunauer-Emmett Teller (BET) method using Quadrasorb SI and silica nanoparticles were pretreated under vacuum condition at $150^{\circ} \mathrm{C}$ for $2 \mathrm{~h}$ before the BET test. HSNs were examined by Fourier Transform Infrared Spectroscopy (FTIR) performed on Nicolet IS10 with a spectra range of $400-4000 \mathrm{~cm}^{-1}$. In order to characterize the organic content and estimate the respective grafting density of the HSNs, a thermogravimetric analysis (TGA) was employed using a TA Q500 and heating at a rate of $10^{\circ} \mathrm{C} / \mathrm{min}$ to $800^{\circ} \mathrm{C}$ under nitrogen.

2.4. Tribological Properties of HSNs. The tribological properties of HSNs with different sizes and organic chains were tested using a MRS-10 four-ball tribometer. The four ball test is a typical method for tribological test. One steel ball rotates against three steel ball under load and steel balls are immersed in lubricant. The $12.7 \mathrm{~mm}$ diameter GCr15 steel bearing ball (grade 4) was obtained from Shanghai Steel Ball Factory 


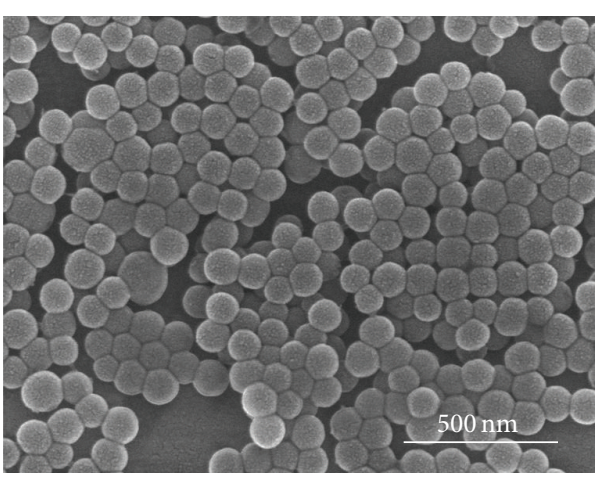

(a)

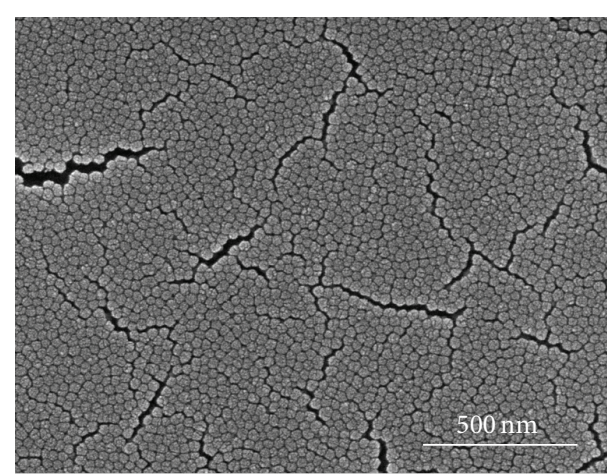

(b)

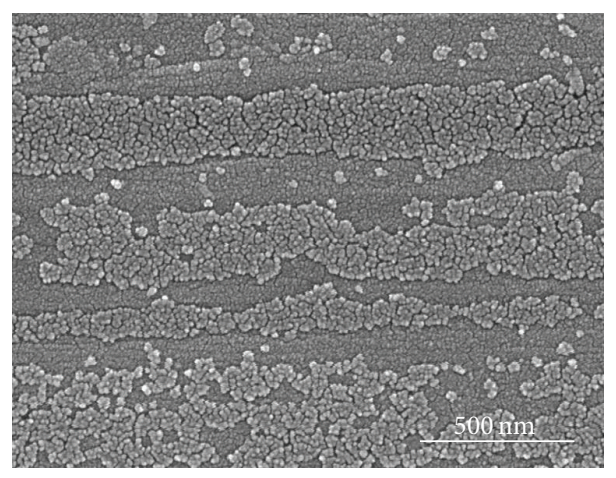

(c)

FIGURE 3: SEM micrograph of (a) $100 \mathrm{~nm}$, (b) $50 \mathrm{~nm}$, and (c) $20 \mathrm{~nm}$ silica nanoparticles.

and cleaned ultrasonically with ethanol before the test. All tests were performed at room temperature under a load of $390 \mathrm{~N}$, rotating speed of $1450 \mathrm{r} / \mathrm{min}$ for $30 \mathrm{~min}$. Tribological tests for different HSNs-PAO lubricants were repeated three times at the same condition. The coefficient of friction (COF) was recorded during the test and the average of COF was calculated. The wear surface of the steel ball was examined by SEM and the wear scar diameter (WSD) was measured.

\section{Results and Discussion}

3.1. Characterization. Silica nanoparticles with three different diameters were synthesized by Stöber method. The $100 \mathrm{~nm}, 50 \mathrm{~nm}$, and $20 \mathrm{~nm}$ silica nanoparticles were shown in Figures 3(a), 3(b), and 3(c), respectively. It can be seen from the figure that silica nanoparticles prepared were pretty round with narrow size distribution. The diameter of $100 \mathrm{~nm}$ nanoparticles was ranging from 93 to $110 \mathrm{~nm}$; for $50 \mathrm{~nm}$ nanoparticles the diameter was at the range of $42 \sim 52 \mathrm{~nm}$ while for $20 \mathrm{~nm}$ one, the diameter was within $15 \sim 22 \mathrm{~nm}$. With the increase of the diameter, both the size uniformity and roundness became better.

BET method was employed to characterize the surface area of USNs. Prior to the experiment, silica nanoparticles were treated under vacuum at $150^{\circ} \mathrm{C}$ for $2 \mathrm{~h}$ and then tested using high purity nitrogen gas. The surface areas of $100 \mathrm{~nm}, 50 \mathrm{~nm}$, and $20 \mathrm{~nm}$ silica nanoparticles were $271 \mathrm{~m}^{2} / \mathrm{g}$, $416 \mathrm{~m}^{2} / \mathrm{g}$, and $547 \mathrm{~m}^{2} / \mathrm{g}$, respectively.

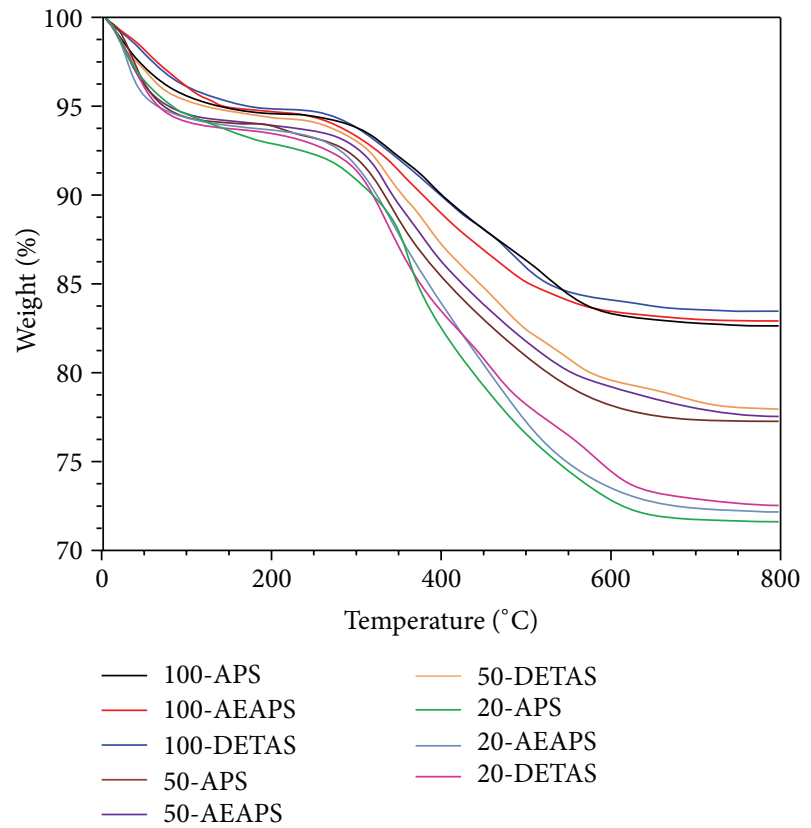

FIgURE 4: Thermogravimetric analysis of HSNs.

The organic content of HSNs was measured using TGA method. The result was shown in Figure 4. It can be seen from the graph that all types of HSNs show the same features. 


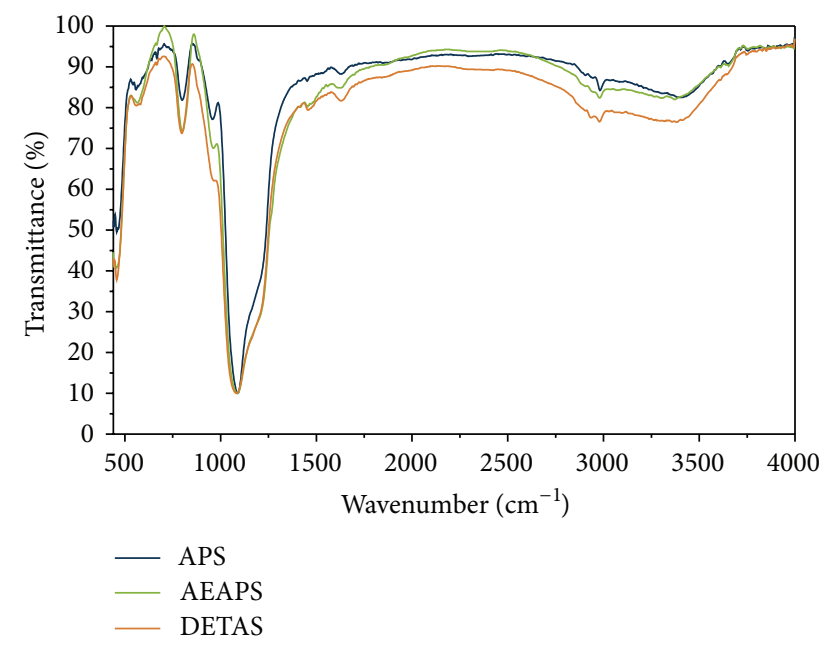

FIgURE 5: The FTIR spectra of HSNs.

Weight loss was found in the beginning of the test because of the evaporation of water remaining in the sample. After a short plateau from 150 to $300^{\circ} \mathrm{C}$, the weight of sample then experienced a sharp decrease when temperature was above $300^{\circ} \mathrm{C}$, which was attributed to the degradation of organic chains grafted on silica nanoparticles. The weight of HSNs stayed stable after $600^{\circ} \mathrm{C}$, indicating that the organic part is fully degraded. As the melting temperature for $\mathrm{SiO}_{2}$ was $1650^{\circ} \mathrm{C}$, the inorganic silica nanoparticle remains at the end of the test. For $100 \mathrm{~nm}$ silica nanoparticles, the organic content was around $11 \%$, for $50 \mathrm{~nm}$ silica nanoparticles, the organic content was around $16 \%$, and for $20 \mathrm{~nm}$ silica nanoparticles, the organic content was about $20 \%$. Based on the testing result of TGA and BET, the grafting density of the silica nanoparticle could be estimated as 1.1 1.2 molecular $/ \mathrm{nm}^{2}$.

The FTIR spectra of HSNs with different organic chains are shown in Figure 5; as can be seen from the graph, all the three spectra of APS, AEAPS, and DETAS show broadband at $1010-1100 \mathrm{~cm}^{-1}$, which could be assigned to $\mathrm{Si}-\mathrm{O}-\mathrm{Si}$ stretching vibration. The peak at $1629 \mathrm{~cm}^{-1}$ in the spectra of DETAS and APS and $1625 \mathrm{~cm}^{-1}$ in the spectrum of AEAPS corresponds to the N-H bending, while the $\mathrm{N}-\mathrm{H}$ stretching vibration appears at $3400 \mathrm{~cm}^{-1}$. The peak which appears at $2981 \mathrm{~cm}^{-1}$ in the spectrum of APS, $2980 \mathrm{~cm}^{-1}$ in AEAPS, and $2978 \mathrm{~cm}^{-1}$ in DETAS is the characteristics of the $\mathrm{C}-\mathrm{H}$ stretching. These results indicate that the APS, AEAPS, and DETAS silanes were grafted on the silica nanoparticle surface successfully. It could also be found from the spectra that the characteristic absorbance of N-H bending for DETAS grafted silica nanoparticles is higher than the other two kinds of silica nanoparticles while that of the APS grafted silica nanoparticles is the lowest one. This agreed well with the feature that the organic chains on DETAS modified HSNs have three amino groups per ligand while APS only has one.

Figure 6 shows the photograph of PAO 100 with USNs ((a) and (c)), PAO 100 with $1 \mathrm{wt} \%$ of $100 \mathrm{~nm}$ HSNs modified with DETAS ((b) and (d)). Figures 6(c) and 6(d) were photographed after 2-month standing of (a) and (b). It could be found from the figure that both HSNs and USNs could form homogeneous solution with PAO 100 when prepared. However, after standing for 2 months, the USNs are sedimentated on the bottom of the bottle while HSNs remain stable and homogeneous.

3.2. Tribology Properties of HSNs. The COF and WSD of $20 \mathrm{~nm}, 50 \mathrm{~nm}$, and $100 \mathrm{~nm}$ HSNs grafted with different organic chains are shown in Figure 7. It could be shown in Figure 7 (a) that, for $20 \mathrm{~nm}$ silica nanoparticles, the best concentration is $1 \mathrm{wt} \%$ and the AEAPT performs the best in three organic chains, the lowest COF is 0.0645 , and lowest WSD is $452 \mu \mathrm{m}$. For $50 \mathrm{~nm}$ silica nanoparticles, the best concentration is around $1 \mathrm{wt} \%$. DETAS modified silica nanoparticles show the best friction reduction and antiwear properties, the best COF is 0.0607 , and the WSD is $301 \mu \mathrm{m}$. For $100 \mathrm{~nm}$ silica nanoparticles, $1 \mathrm{wt} \%$ DETAS modified silica nanoparticles exhibited an optimum reduction in WSD and COF, of which the best COF is 0.05692 and WSD is $305 \mu \mathrm{m}$. It could be found from the graph that the tribological performance of HSNs generally becomes better with the increase of nanoparticle size. DETAS modified HSNs have overall better antiwear and friction reduction performance in the three types of organic silanes.

It could be found from the histogram that the COF and WSD experienced significant reduction when concentration increased from 0.5 to $1 \mathrm{wt} \%$. That may be due to insufficient nanoparticles between two metal surfaces at low concentration, which lead to inefficient protect of metal surfaces. Meanwhile, when the concentration increased from 2 to $4 \mathrm{wt} \%$, the COF and WSD showed remarkable increment. This may be caused by excessive nanoparticles at high concentration, which result in congestion of nanoparticles and scratching the metal surface (jamming effect).

Figure 8 shows the COF versus time of PAO 100 and PAO 100 with $100 \mathrm{~nm}$ HSNs. Typical COF curves of $100 \mathrm{~nm}$ HSNs (the COF curve of different HSNs at their best concentration) were chosen. It could be found from the figure that the COF of PAO 100 is around 0.065 at the beginning and experiences a short stable period after the test started. The COF then increases drastically from 0.065 to about 0.09 around $500 \mathrm{~s}$, followed by rising with fluctuations to 0.11 . For PAO with $1 \mathrm{wt} \% 100 \mathrm{~nm}$ DETAS modified HSNs, the COF starts at 0.06 and decreases to about 0.056 around $200 \mathrm{~s}$ and then remains steady in the rest of the test. For AEAPS, the COF rises sharply from 0.063 to 0.10 around $300 \mathrm{~s}$ and then drops slowly; at the end of the test the COF is about 0.062 . For APS, the COF starts at 0.60 and then rises slowly with fluctuation; at the end of the test, the COF reaches 0.08 . It could be found from the graph that, with the same diameter of silica nanoparticles, DETAS modified nanoparticles perform the best friction reduction properties which has lowest COF and smoothest curve.

Figure 9 shows the COF versus time of PAO 100 and HSNs-PAO with different diameters of DETAS modified HSNs. Typical COF curve of DETAS modified HSNs (1 wt $\%$ 


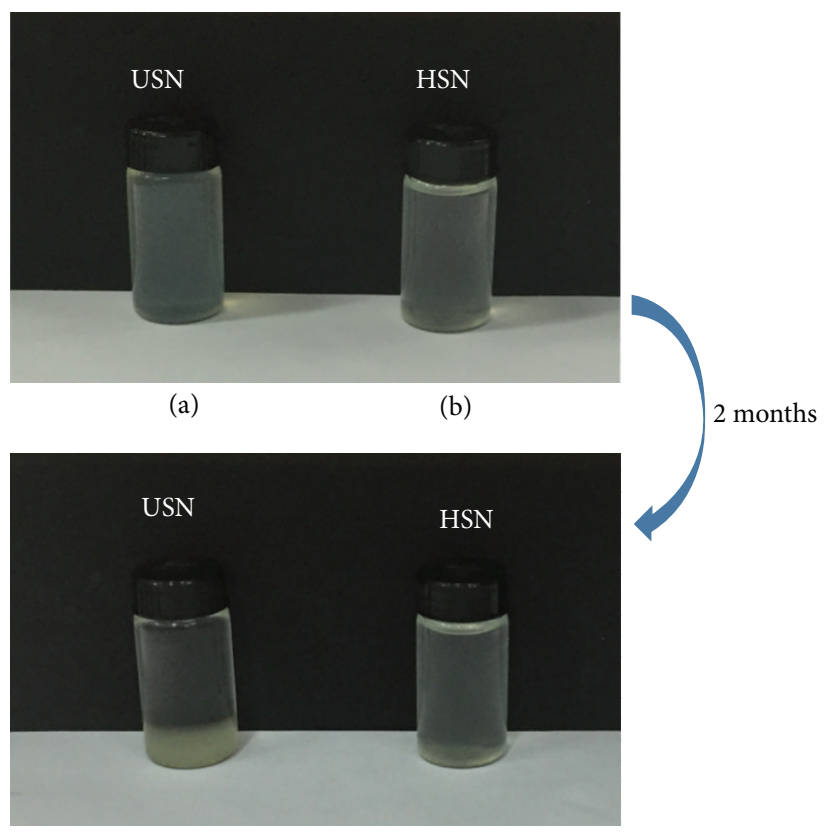

(c)

(d)

FIgUre 6: Photograph of (a, c) PAO 100 with USNs and (b, d) PAO 100 with HSNs. PAO 100 with silica nanoparticles shown in (c) and (d) were photographed after 2-month standing.

of DETAS modified $20 \mathrm{~nm}, 50 \mathrm{~nm}$, and $100 \mathrm{~nm}$ HSNs) was chosen. As can be seen from the graph, $100 \mathrm{~nm}$ and $50 \mathrm{~nm}$ silica nanoparticles show the similar trend which remains steady and slightly decreases during the whole test while the COF of $50 \mathrm{~nm}$ HSNs is higher than the $100 \mathrm{~nm}$ for about 0.05 . The COF of $20 \mathrm{~nm}$ HSNs rises with fluctuation and increases to 0.08 at the end of the test. It could be found from the graph that, with the same modifier, $100 \mathrm{~nm}$ HSNs perform the best tribological property while $20 \mathrm{~nm}$ ones perform the worst, which met well with the findings in Figure 7. It could be found from the experiment that adding HSNs to the PAO 100 enhances the friction reduction properties greatly which could optimally reduce the COF by $40 \%$.

The antiwear and friction reduction mechanisms of nanoparticles as additives in lubricant are shown in Figure 10. When nanoparticles were taken into the contacting area by lubricants, they would fill in the microgrooves on the metal surface (mending effect) and polish the rough metal surfaces (polishing effect). Both of them could reduce the roughness of the surface, thereby reducing friction and wear. Some nanoparticles interact with the metal surface and form transfer films on it, which could protect the surface and reduce the friction (protecting effect). For rolling effect, nanoparticles are likely to support the metal surfaces and prevent them from contacting each other and rolling between two metal surfaces like bearing ball in bearing.

It could be found from the experiment result that, with the increase of nanoparticle size, the WSD and COF overall decrease. This may be due to the rolling effect and mending effect of nanoparticles. Silica nanoparticles with larger diameter could support the metal surface and prevent the contact more effectively than smaller ones. Meanwhile, with better roundness, larger nanoparticles might roll better between two surfaces and reduce the abrasive effect of the nanoparticle. With the same concentration, larger nanoparticles might fill in the microgrooves more effectively than smaller one, which would lead to better surface roughness and reduce the friction and wear. DETAS modified HSNs show overall better antiwear and friction reduction properties than other two types of HSNs while APS type shows the worst, which may be due to the organic chain length and amino groups. Silica nanoparticles may aggregate by the hydrogen bond between primary functional amino groups, which would lead to bad dispersion and cause three-body abrasion. With a longer organic chain, the DETAS grafted silica nanoparticles could prevent the aggregation of nanoparticles more effectively and keep the dispersity of nanoparticles. Meanwhile, increasing numbers of the amino groups could enhance the absorption of nanoparticles on the metal surface, which could be attributed to the physical adsorption (the chelation effect and polar adsorption of amino group) and the chemical binding (the hemilability of amine-metal bond). With more HSNs adsorbed on the metal surface, HSNs would have more opportunities to interact with metal surfaces; as a result, all of the four effects of nanoparticles mentioned above (mending, polishing, rolling, and protecting effect) would be enhanced. It should be noted here that the COF curve of HSNs-PAO keeps slightly decreasing after it reaches the steady state. This may be due to the polishing effect and protecting effect of nanoparticles which improves the surface roughness of steel balls in the beginning and forming of transfer film when COF reaches steady state. 


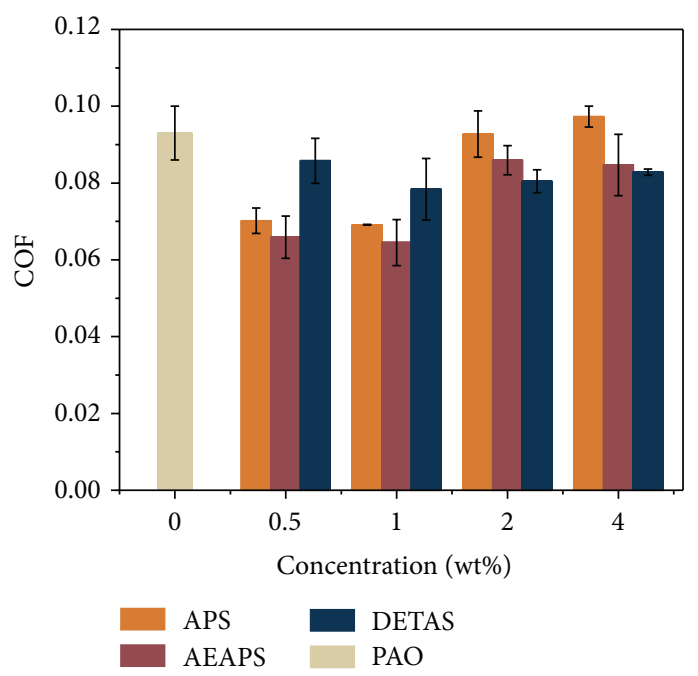

(a)

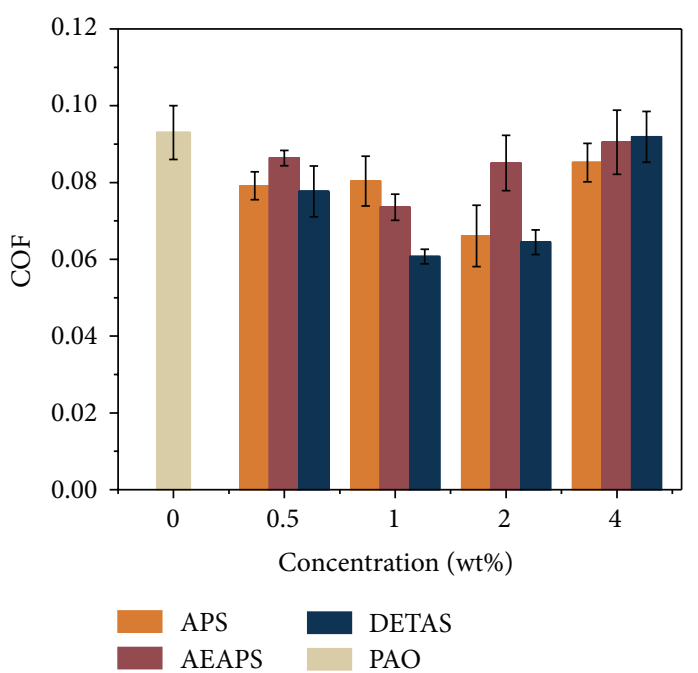

(c)

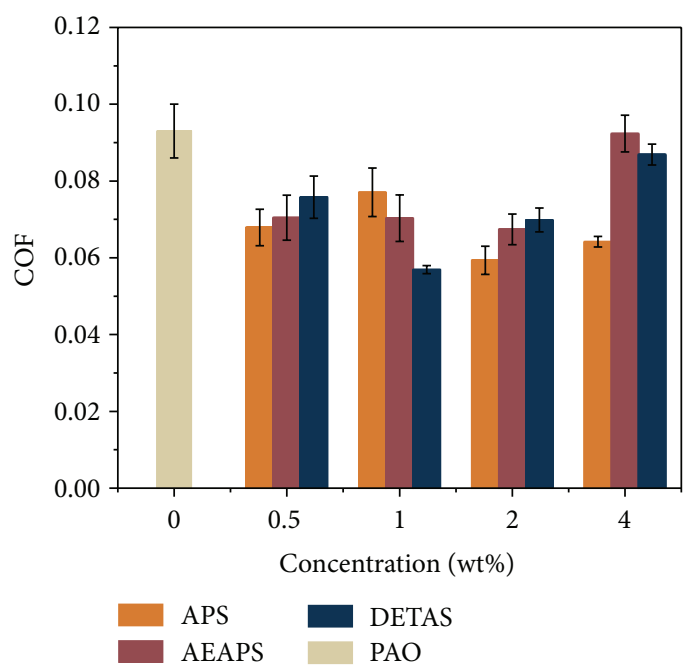

(e)

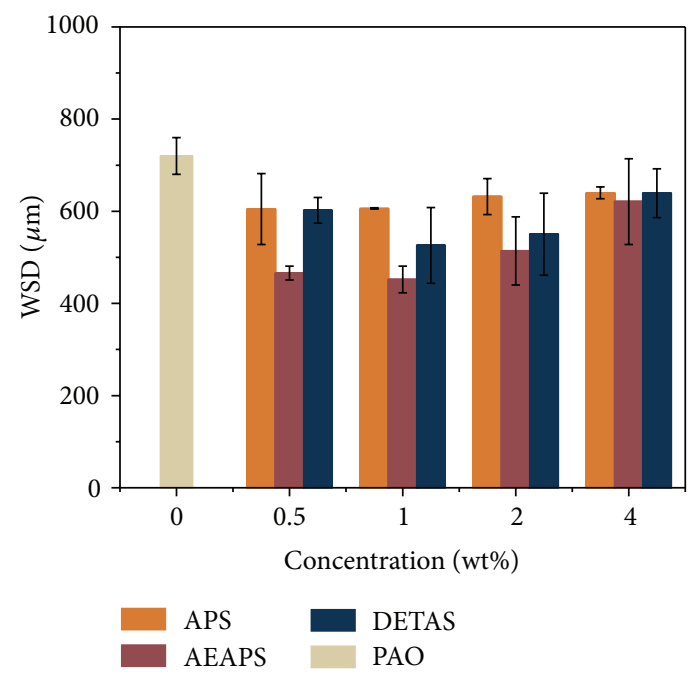

(b)

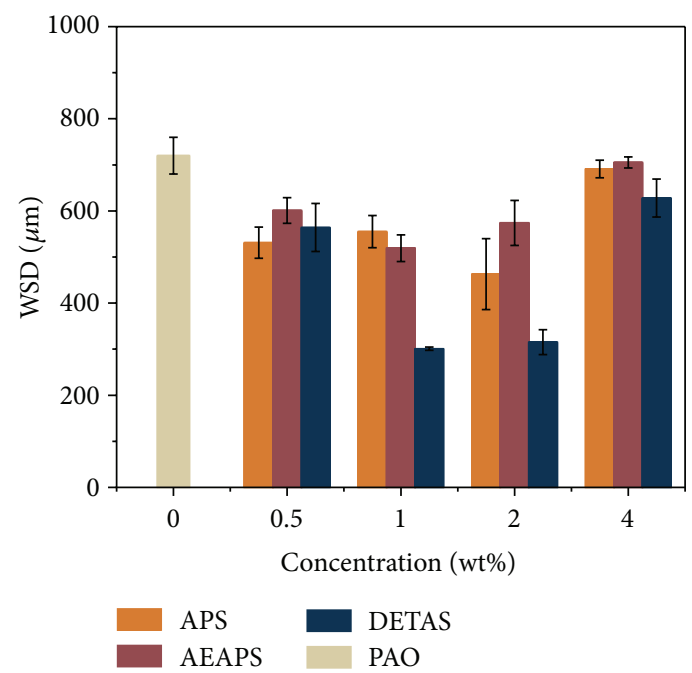

(d)

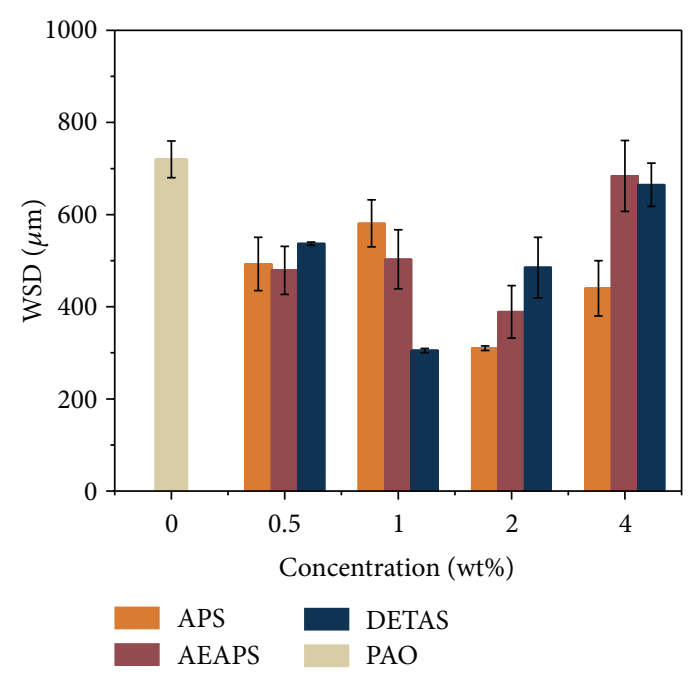

(f)

Figure 7: COF and WSD of (a, b) $20 \mathrm{~nm},(\mathrm{c}, \mathrm{d}) 50 \mathrm{~nm}$, and (e, f) $100 \mathrm{~nm}$ HSNs-PAO lubricants. 


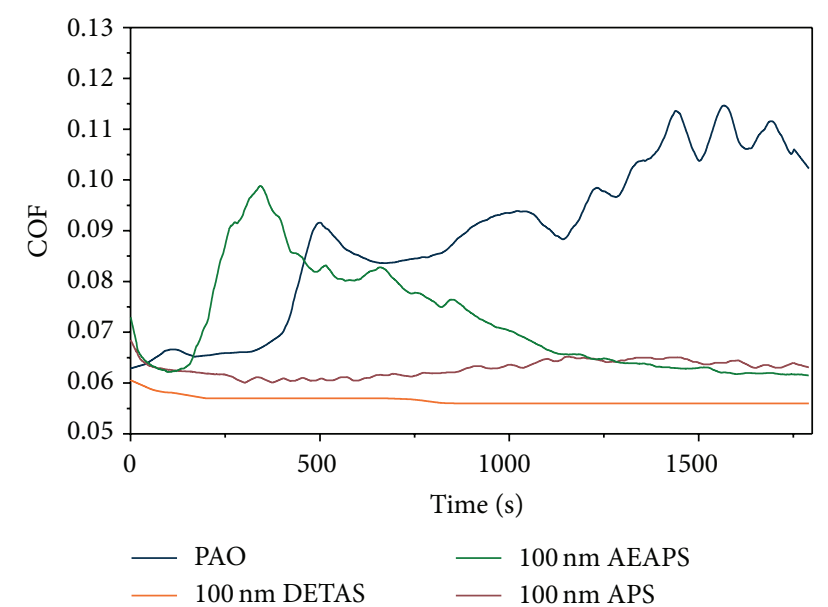

Figure 8: The COF versus time of PAO 100 and PAO 100 with $100 \mathrm{~nm}$ HSNs grafted with different organic chains.

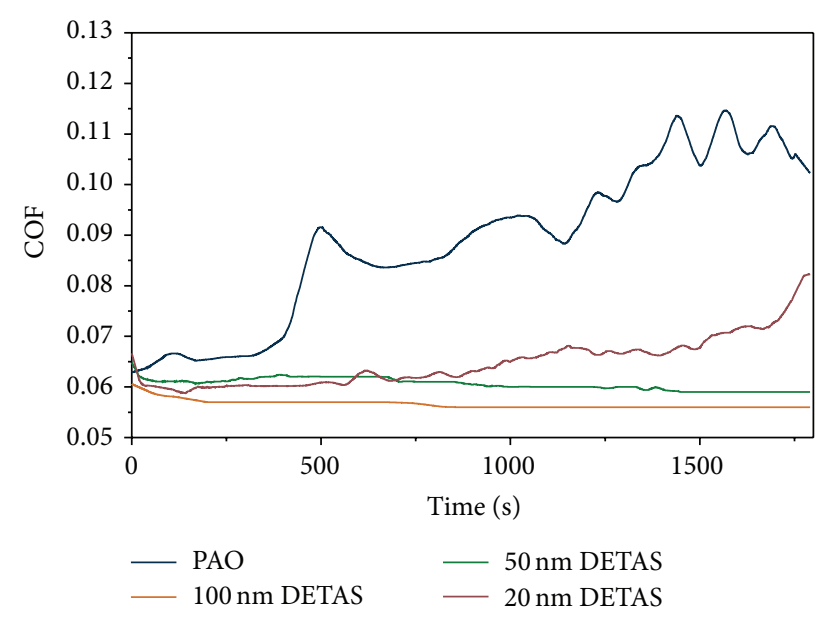

Figure 9: The COF versus time of PAO 100 and HSNs-PAO with different size DETAS HSNs.

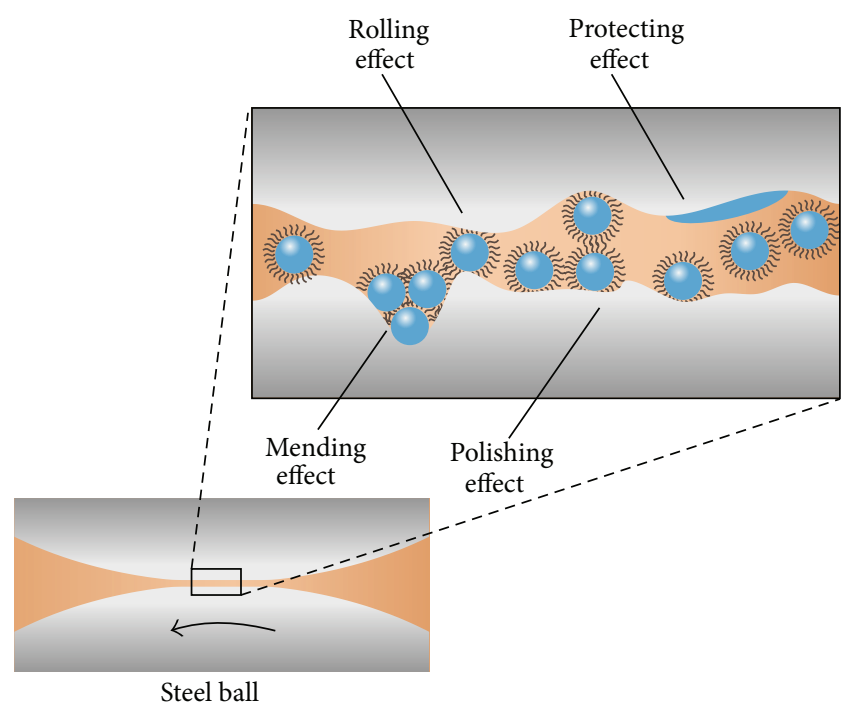

FIGURE 10: The schematic diagram of the lubrication mechanism of silica nanoparticle.

\section{Conclusions}

In this study, HSNs with different size and organic chains were synthesized and dispersed into PAO lubricant. The HSNs were characterized by SEM, TEM, BET, FTIR, and TGA. The result shows that organic chains were densely grafted on the nanoparticles. Tribological properties of HSNs were tested using a four-ball tribometer; the wear mechanism was analyzed. Using the four-step preparation method described in this paper, silica nanoparticles grafted with amino-functional organic chains could disperse in PAO 100 homogeneously and remain stable for long time. After adding amino functionalized HSNs, the COF optimally reduces for $40 \%$ and WSD reduces more than $60 \%$ comparing to pure PAO 100. The COF and WSD increase with the increase of diameter; silica nanoparticle with $100 \mathrm{~nm}$ diameter performs best in the tribological test while $20 \mathrm{~nm}$ diameter silica nanoparticle performs the worst, which could be attributed to the better rolling effect that could support two-metal surface. HSNs grafted with DETAS show the best antiwear and friction reduction properties among the three-silane test in this paper, which suggested that longer organic chains and more amino group would enhance the tribological performance of HSNs due to their better dispersion and adsorption on the metal surface.

\section{Conflict of Interests}

The authors declare that there is no conflict of interests regarding the publication of this paper.

\section{Acknowledgments}

The authors wish to thank Professor Donald Koch, Professor Lynden Archer, and Rahul Mangal, Chemical and Biological Engineering, Cornell University, for assisting with the synthesis of HSNs and comments for experiments.

\section{References}

[1] W.-L.-J. Hasi, X. Lin, X.-T. Lou et al., "Chloride ion-assisted self-assembly of silver nanoparticles on filter paper as SERS substrate," Applied Physics A, vol. 118, no. 3, pp. 799-807, 2015.

[2] A. Bitar, N. M. Ahmad, H. Fessi, and A. Elaissari, "Silica-based nanoparticles for biomedical applications," Drug Discovery Today, vol. 17, no. 19-20, pp. 1147-1154, 2012.

[3] M. Ratoi, P. H. M. Hoet, A. Crossley, and P. Dobson, "Impact of lung surfactant on wettability and cytotoxicity of nanoparticles," RSC Advances, vol. 4, no. 39, pp. 20573-20581, 2014.

[4] J. L. Vivero-Escoto, I. I. Slowing, B. G. Trewyn, and V. S.-Y. Lin, "Mesoporous silica nanoparticles for intracellular controlled drug delivery," Small, vol. 6, no. 18, pp. 1952-1967, 2010.

[5] S. Ingole, A. Charanpahari, A. Kakade, S. S. Umare, D. V. Bhatt, and J. Menghani, "Tribological behavior of nano $\mathrm{TiO}_{2}$ as an additive in base oil," Wear, vol. 301, no. 1-2, pp. 776-785, 2013.

[6] Y. Gao, G. Chen, Y. Oli, Z. Zhang, and Q. Xue, "Study on tribological properties of oleic acid-modified $\mathrm{TiO}_{2}$ nanoparticle in water," Wear, vol. 252, no. 5-6, pp. 454-458, 2002.

[7] B.-S. Zhang, B.-S. Xu, Y. Xu, F. Gao, P.-J. Shi, and Y.-X. $\mathrm{Wu}$, "CU nanoparticles effect on the tribological properties 
of hydrosilicate powders as lubricant additive for steel-steel contacts," Tribology International, vol. 44, no. 7-8, pp. 878-886, 2011.

[8] Y. Zhang, H. Tang, X. Ji et al., "Synthesis of reduced graphene oxide/ $\mathrm{Cu}$ nanoparticle composites and their tribological properties," RSC Advances, vol. 3, no. 48, pp. 26086-26093, 2013.

[9] A. Hernandez Battez, J. E. Fernandez Rico, A. Navas Arias, J. L. Viesca Rodriguez, R. Chou Rodriguez, and J. M. Diaz Fernandez, "The tribological behaviour of $\mathrm{ZnO}$ nanoparticles as an additive to PAO6," Wear, vol. 261, no. 3-4, pp. 256-263, 2006.

[10] J. Padgurskas, R. Rukuiza, I. Prosyčevas, and R. Kreivaitis, "Tribological properties of lubricant additives of $\mathrm{Fe}, \mathrm{Cu}$ and Co nanoparticles," Tribology International, vol. 60, pp. 224-232, 2013.

[11] S. Zhang, L. Hu, D. Feng, and H. Wang, "Anti-wear and frictionreduction mechanism of $\mathrm{Sn}$ and Fe nanoparticles as additives of multialkylated cyclopentanes under vacuum condition," Vacuum, vol. 87, pp. 75-80, 2013.

[12] M. Ratoi, V. B. Niste, and J. Zekonyte, "WS 2 nanoparticlespotential replacement for ZDDP and friction modifier additives," RSC Advances, vol. 4, no. 41, pp. 21238-21245, 2014.

[13] Z.-Z. Wang, P. Gu, X.-P. Wu, H. Zhang, Z. Zhang, and M. Y. M. Chiang, "Micro/nano-wear studies on epoxy/silica nanocomposites," Composites Science and Technology, vol. 79, pp. 49-57, 2013.

[14] H.-S. Jung, D.-S. Moon, and J.-K. Lee, "Quantitative analysis and efficient surface modification of silica nanoparticles," Journal of Nanomaterials, vol. 2012, Article ID 593471, 8 pages, 2012.

[15] B. Sarkar, V. Venugopal, A. M. Bodratti, M. Tsianou, and P. Alexandridis, "Nanoparticle surface modification by amphiphilic polymers in aqueous media: role of polar organic solvents," Journal of Colloid and Interface Science, vol. 397, pp. $1-8,2013$.

[16] N. Plumeré, A. Ruff, B. Speiser, V. Feldmann, and H. A. Mayer, "Stöber silica particles as basis for redox modifications: particle shape, size, polydispersity, and porosity," Journal of Colloid and Interface Science, vol. 368, no. 1, pp. 208-219, 2012.

[17] R. P. Bagwe, L. R. Hilliard, and W. Tan, "Surface modification of silica nanoparticles to reduce aggregation and nonspecific binding," Langmuir, vol. 22, no. 9, pp. 4357-4362, 2006.

[18] X. Li, Z. Cao, Z. Zhang, and H. Dang, "Surface-modification in situ of nano- $\mathrm{SiO}_{2}$ and its structure and tribological properties," Applied Surface Science, vol. 252, no. 22, pp. 7856-7861, 2006.

[19] E. Amerio, P. Fabbri, G. Malucelli, M. Messori, M. Sangermano, and R. Taurino, "Scratch resistance of nano-silica reinforced acrylic coatings," Progress in Organic Coatings, vol. 62, no. 2, pp. 129-133, 2008.

[20] A.-C. Hervé, J.-J. Yaouanc, J.-C. Clément, H. des Abbayes, and L. Toupet, "Hemilability of the primary amine-metal bond in polyamine-(group 6) metal carbonyl complexes," Journal of Organometallic Chemistry, vol. 664, no. 1-2, pp. 214-222, 2002.

[21] B. Yu, L. Qian, J. Yu, and Z. Zhou, "Effects of tail group and chain length on the tribological behaviors of self-assembled dual-layer films in atmosphere and in vacuum," Tribology Letters, vol. 34, no. 1, pp. 1-10, 2009.

[22] T. Jiang, T. Kuila, N. H. Kim, B.-C. Ku, and J. H. Lee, "Enhanced mechanical properties of silanized silica nanoparticle attached graphene oxide/epoxy composites," Composites Science and Technology, vol. 79, pp. 115-125, 2013.
[23] S. Song, R. Chu, J. Zhou, S. Yang, and J. Zhang, "Formation and tribology study of amide-containing stratified self-assembled monolayers: influences of the underlayer structure," Journal of Physical Chemistry C, vol. 112, no. 10, pp. 3805-3810, 2008.

[24] J. Y. Seo and M. Han, "Multi-functional hybrid coatings containing silica nanoparticles and anti-corrosive acrylate monomer for scratch and corrosion resistance," Nanotechnology, vol. 22, no. 2, Article ID 025601, 2011.

[25] D. Kim and L. A. Archer, "Nanoscale organic-inorganic hybrid lubricants," Langmuir, vol. 27, no. 6, pp. 3083-3094, 2011.

[26] G. Zhang, R. Sebastian, T. Burkhart, and K. Friedrich, "Role of monodispersed nanoparticles on the tribological behavior of conventional epoxy composites filled with carbon fibers and graphite lubricants," Wear, vol. 292-293, pp. 176-187, 2012.

[27] D. X. Peng, Y. Kang, R. M. Hwang, S. S. Shyr, and Y. P. Chang, "Tribological properties of diamond and $\mathrm{SiO}_{2}$ nanoparticles added in paraffin," Tribology International, vol. 42, no. 6, pp. 911917, 2009.

[28] W. Stöber, A. Fink, and E. Bohn, "Controlled growth of monodisperse silica spheres in the micron size range," Journal of Colloid and Interface Science, vol. 26, no. 1, pp. 62-69, 1968. 

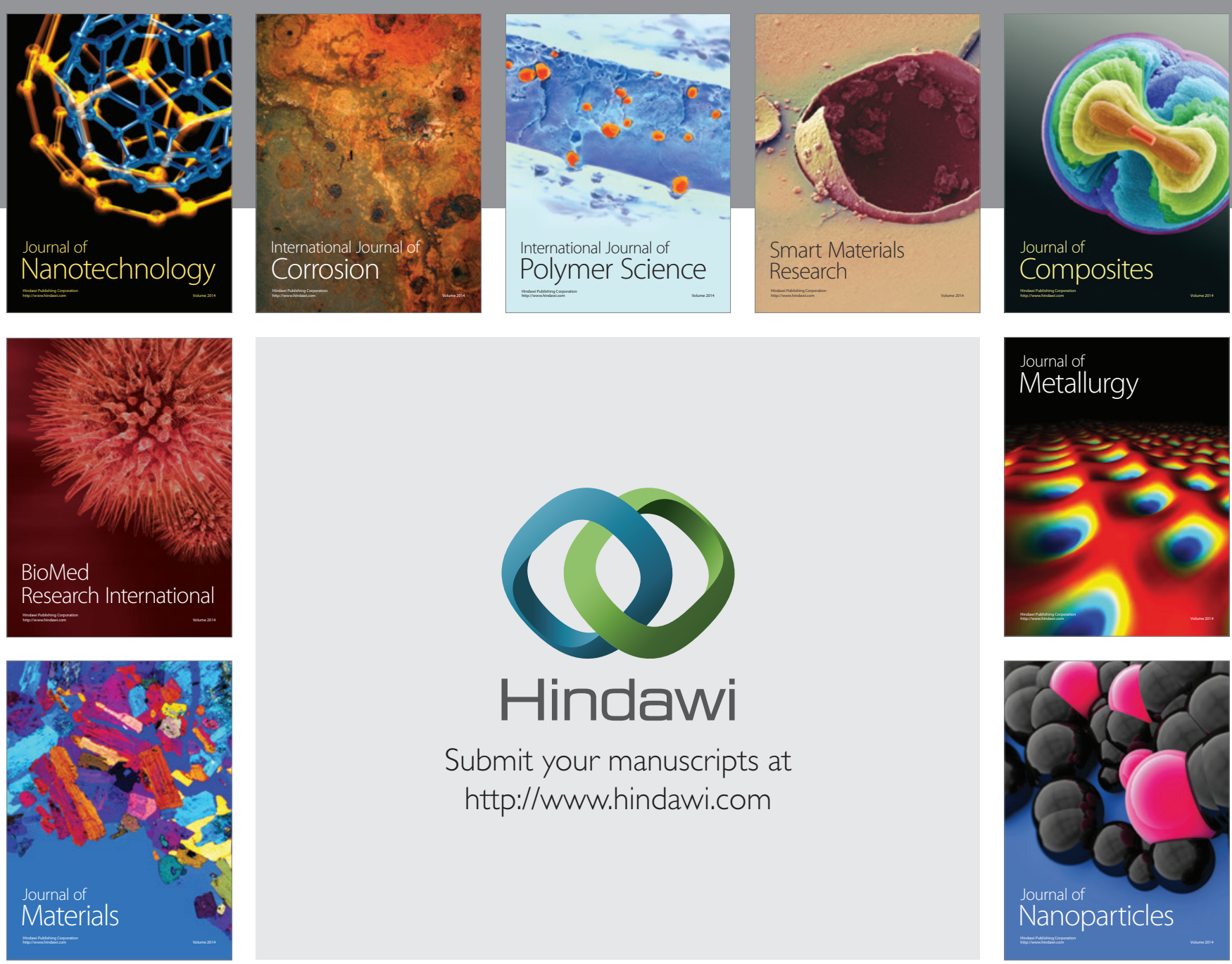

Submit your manuscripts at http://www.hindawi.com
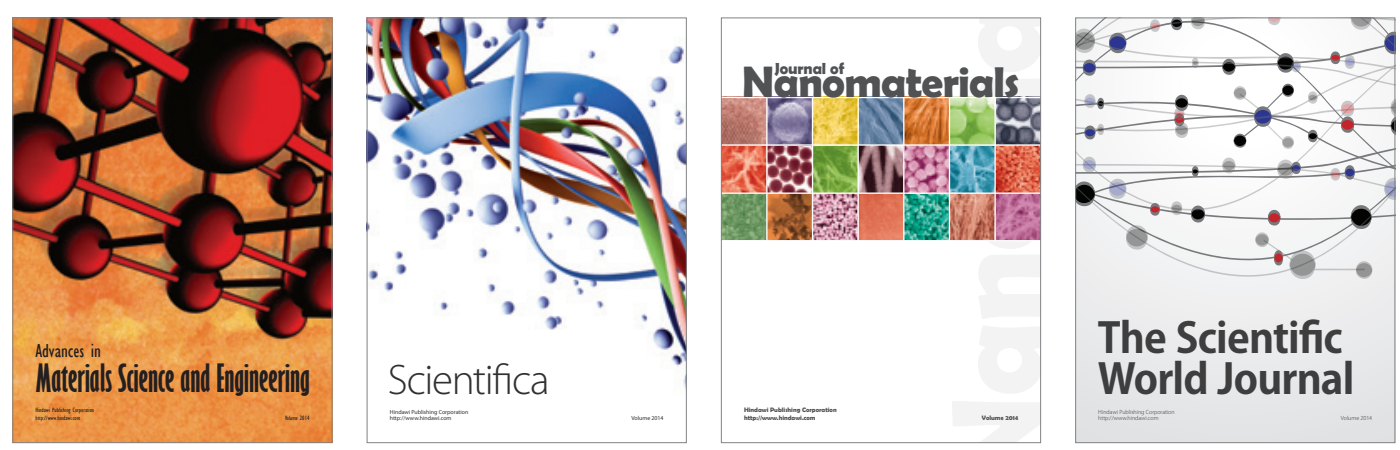

\section{The Scientific World Journal}
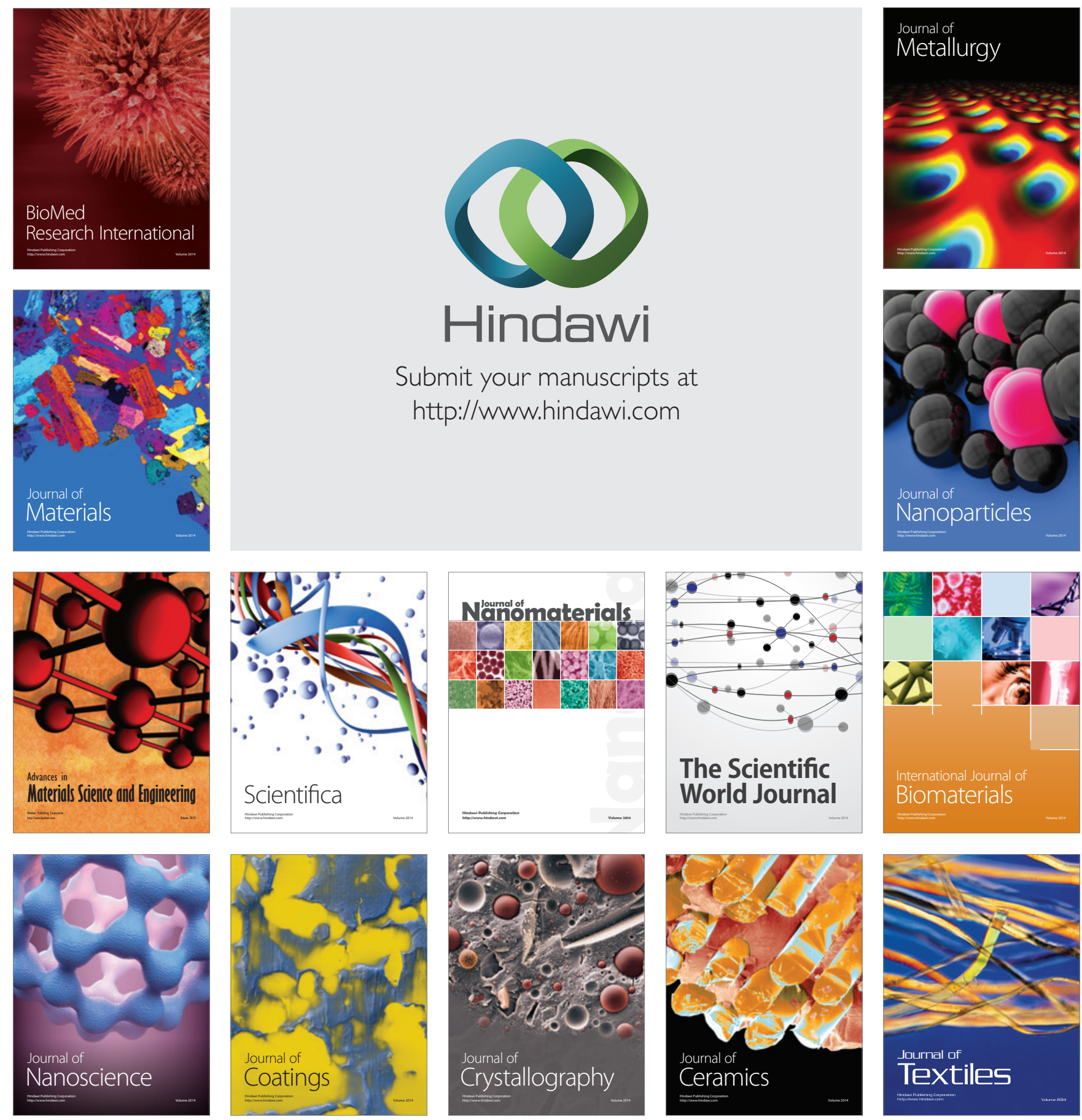